\title{
Some fixed point theorems for $\alpha$ - $\varphi$-contractive mappings in cone 2-metric spaces
}

\author{
V. H. Badshah, P. Bhagat, S. Shukla
}

\begin{abstract}
In this paper, we introduce the notion of $\alpha$-admissible mappings in the setting of cone 2-metric spaces over Banach algebras. Some existence and uniqueness results of the fixed point of an $\alpha-\varphi$-contractive mapping in cone 2 -metric spaces are proved. Some examples are provided which illustrate the results proved herein.
\end{abstract}

Keywords: Fixed point; cone 2-metric; comparison function; $\alpha$ - $\varphi$-contractive mapping.

\section{Introduction and Preliminaries}

Let $X$ be a nonempty set and $d: X \times X \times X \rightarrow \mathbb{R}^{+}$(the set of nonnegative real numbers) be any function satisfying the following properties:

(1) $d(x, y, z)=0$ if at least two of $x, y, z$ are equal;

(2) $d(x, y, z)=d(p(x, y, z))$ for all $x, y, z \in X$, where $p(x, y, z)$ denotes all the permutations of $x, y, z$;

(3) $d(x, y, z) \leq d(x, y, w)+d(x, w, z)+d(w, y, z)$ for all $x, y, z, w \in X$.

Then $d$ is called a 2-metric on $X$ and the pair $(X, d)$ is called a 2-metric space. Gähler $[10,11,12]$ first introduced the notion of 2 -metric spaces as a generalization of ordinary metric spaces. In the papers [10, 11, 12] Gähler studied several properties of such spaces. On the other hand, Huang and Zhang [9] introduced the notion of cone metric spaces. They used the ordered Banach spaces as a codomain of the metric defined on a nonempty set and proved some basic versions of fixed point theorems for contractive mappings on such spaces. Liu and $\mathrm{Xu}[6]$ defined the cone metric spaces over Banach algebras and proved

Manuscript received May 12, 2016; accepted September 18, 2016.

Viquar Hussain Badshah is with the School of Studies in Mathematics, Vikram University, Ujjain, India; Prakash Bhagat and Satish Shukla are with the Department of Applied Mathematics, Shri Vaishnav Institute of Technology \& Science, Indore, India 
the fixed point results for the contractive mappings with vector contractive constants. The drawback of fixed point theorems in cone metric spaces in the sense of Huang and Zhang [9] and the motivation for the work of Liu and Xu [6] can be found in [5, 17, 18, 19]. Singh et al. [3] combined the concepts of cone metric and 2-metric, and introduced a new type of spaces called cone 2-metric spaces. They also proved a fixed point result assuming that the underlying cone is normal. Very recently, Wang et al. [15] improved the result of Singh et al. [3] by introducing the cone 2-metric spaces over Banach algebra.

In 2003, Ran and Reurings [1] proved an analogue of Banach's fixed point theorem in partially ordered sets endowed with a metric. The results of Ran and Reurings [1] were further investigated by Nieto and Rodríguez-López. Samet et al. [2] introduced the study of a new class of mappings called $\alpha$-admissible mapping, on metric spaces and generalized the results of Ran and Reurings [1] and Nieto and Rodríguez-López. They established some fixed point theorems for $\alpha-\psi$-contractive type mappings. In this paper, we introduce the the notion of $\alpha$-admissible mappings in the setting of cone 2-metric spaces over Banach algebra, and prove some fixed point results for a new class of mappings called $\alpha-\varphi$-contractive mapping. Our results generalize and extend the results of Samet et al. [2] in cone 2-metric spaces. Therefore, we obtain the cone 2-metric versions of the results of Ran and Reurings [1] and Nieto and Rodríguez-López. Some examples are provided which confirm the significance of our results.

First, we recall some definitions and properties which will be used throughout the paper.

Let $A$ always be a real Banach algebra with a multiplicative unit $e$, that is, $e x=x e=x$ for all $x \in A$. An element $x \in A$ is said to be invertible if there is an inverse element $y \in A$ such that $x y=y x=e$. The inverse of $x$ is denoted by $x^{-1}$ (see [16]).

The following proposition can be found, e.g., in [16].

Proposition 1.1. Let $A$ be a Banach algebra with the unit $e$, and $x \in A$. If the spectral radius $\rho(x)$ of $x$ is less than 1 , that is,

$$
\rho(x)=\lim _{n \rightarrow \infty}\left\|x^{n}\right\|^{1 / n}=\inf _{n \geq 1}\left\|x^{n}\right\|^{1 / n}<1
$$

then $e-x$ is invertible. Actually,

$$
(e-x)^{-1}=\sum_{i=0}^{\infty} x^{i}
$$

A subset $P$ of $A$ is called a cone if:

(1) $P$ is nonempty closed and $\{\theta, e\} \subset P$;

(2) $\alpha P+\beta P \subset P$ for all nonnegative real numbers $\alpha, \beta$;

(3) $P^{2}=P P \subset P$; 
(4) $P \cap(-P)=\{\theta\}$

where $\theta$ and $e$ are respectively the zero vector and unit of $A$.

Given a cone $P \subset A$, we define a partial ordering $\preceq$ in $A$ with respect to $P$ by $x \preceq y$ (or equivalently $y \succeq x$ ) if and only if $y-x \in P$. We shall write $x \prec y$ (or equivalently $y \succ x$ ) to indicate that $x \preceq y$ but $x \neq y$, while $x \ll y$ (or equivalently $y \gg x$ ) will stand for $y-x \in \operatorname{int} P$, where int $P$ denotes the interior of $P$.

The cone is called normal if there exists a number $K>0$ such that for all $x, y \in P$

$$
x \preceq y \Longrightarrow\|x\| \leq K\|y\| .
$$

The least number $K$ satisfying the above inequality is called the normal constant of $P$. The cone $P$ is called solid if int $P \neq \emptyset$.

In the following, we always assume that the cone $P$ is solid cone in Banach algebra $A$ and $\preceq$ is partial ordering with respect to $P$.

Proposition 1.2 ([14]). Let $P$ be a cone in a Banach algebra $A, a \in P$ and $b, c \in A$ are such that $b \preceq c$, then $a b \preceq a c$.

Lemma 1.3 ([13, 20]). Let A be a Banach algebra with a solid cone P. Then:

(a) If $a \preceq \lambda a$ with $a \in P$ and $0 \leq \lambda<1$, then $a=\theta$.

(b) If $\theta \preceq u \ll c$ for each $\theta \ll c$, then $u=\theta$.

(c) If $\left\|x_{n}\right\| \rightarrow 0$ as $n \rightarrow \infty$, then for any $\theta \ll c$, there exists $n_{0} \in \mathbb{N}$ such that, $x_{n} \ll c$ for all $n>n_{0}$.

Remark 1.4 ([14]). If $\rho(x)<1$ then $\left\|x^{n}\right\| \rightarrow 0$ as $n \rightarrow \infty$.

Definition 1.5 ([3,15]). Let $X$ be a nonempty set. Suppose the mapping $d: X \times X \times X \rightarrow P$ satisfies:

1. $\theta \preceq d(x, y, z)$ for all $x, y, z \in X$, and $d(x, y, z)=\theta$ if and only if at least two of $x, y, z$ are equal.

2. $d(x, y, z)=d(p(x, y, z))$ for all $x, y, z \in X$, where $p(x, y, z)$ denotes all the permutations of $x, y, z$.

3. $d(x, y, z) \preceq d(x, y, w)+d(x, w, z)+d(w, y, z)$ for all $x, y, z, w \in X$.

Then $d$ is called a cone 2-metric on $X$, and $(X, d)$ will be called a cone 2-metric space over Banach algebras A. Cone 2-metric space will be called normal, if the cone $P$ is normal cone. 
Example 1.6. Let $X=[0, \infty), A=C^{1}([0,1], \mathbb{R})$ be the Banach algebra with pointwise multiplication and the norm defined by $\|x(t)\|=\|x(t)\|_{\infty}+\left\|x^{\prime}(t)\right\|_{\infty}$. Let $P=\{x(t) \in$ $C^{1}([0,1], \mathbb{R}): x(t) \geq 0$ for all $\left.t \in[0,1]\right\}$, then $P$ is a solid (non-normal) cone in $A$. Define a function $d: X \times X \times X \rightarrow P$ by

$$
d(x, y, z)= \begin{cases}(x y+y z+z x) e^{t}, & \text { if } x \neq y \neq z \\ 0, & \text { otherwise. }\end{cases}
$$

Then $(X, d)$ is a cone 2-metric space over Banach algebras $A$.

Definition 1.7 ([3]). Let $(X, d)$ be a cone 2-metric space with a solid cone $P$ in Banach algebra $A$. Let $\left\{x_{n}\right\}$ be a sequence in $X$ and $x \in X$. If for every $c \in A$ with $\theta \ll c$ (that is, $c \in$ intP) there exists $n_{0} \in \mathbb{N}$ such that $d\left(x_{n}, x, a\right) \ll c$ for all $a \in X$ and for all $n>n_{0}$, then $\left\{x_{n}\right\}$ is said to be convergent and converges to $x$. We denote it by $\lim _{n \rightarrow \infty} x_{n}=x$ or $x_{n} \rightarrow x$ as $n \rightarrow \infty$.

Remark 1.8. The limit of a convergent sequence in a cone 2-metric space with solid cone is unique. For instance, if a sequence $\left\{x_{n}\right\} \subset X$ has two distinct limits say $x, y \in X$, then for every $c \in A$ with $\theta \ll c$ there exists $n_{0} \in X$ such that $d\left(x_{n}, x, a_{1}\right) \ll c / 3$ and $d\left(x_{n}, y, a_{2}\right) \ll c / 3$ for all $a_{1}, a_{2} \in X$ and for all $n>n_{0}$. Therefore by Lemma 1.3 we have

$$
d(x, y, a) \preceq d\left(x, y, x_{n}\right)+d\left(x, x_{n}, a\right)+d\left(x_{n}, y, a\right) \ll \frac{c}{3}+\frac{c}{3}+\frac{c}{3}=c
$$

for all $a \in X$ and for all $n>n_{0}$. Therefore, $x=y$, which completes the proof.

Definition 1.9 ([3]). Let $(X, d)$ be a cone 2-metric space with a solid cone $P$ in Banach algebra $A$. Let $\left\{x_{n}\right\}$ be a sequence in $X$ and $x \in X$. If for every $c \in A$ with $\theta \ll c$ there exists $n_{0} \in \mathbb{N}$ such that $d\left(x_{n}, x_{m}, a\right) \ll c$ for all $a \in X$ and for all $n, m>n_{0}$, then $\left\{x_{n}\right\}$ is said to be a Cauchy sequence.

Definition 1.10 ([3]). Let $(X, d)$ be a cone 2-metric space, if every Cauchy sequence is convergent in $X$, then $X$ is said to be a complete cone 2-metric space.

Definition 1.11 ([4]). Let $P$ be a cone in Banach algebra A. A nondecreasing function $\varphi: P \rightarrow P$ is called a comparison function if it satisfies:

(i) $\varphi(\theta)=\theta$ and $\theta \prec \varphi(x) \prec x$ for all $x \in P \backslash\{\theta\}$.

(ii) If $x \in$ intP then $x-\varphi(x) \in \operatorname{intP}$.

(iii) $\lim _{n \rightarrow \infty} \varphi^{n}(x)=\theta$ for all $x \in P \backslash\{\theta\}$.

Example 1.12. Let $A=\mathbb{R}^{2}$ be the Banach algebra with Euclidian norm and multiplication defined by $\left(x_{1}, x_{2}\right) \cdot\left(y_{1}, y_{2}\right)=\left(x_{1} y_{1}, x_{1} y_{2}+x_{2} y_{1}\right)$. Let $P=\left\{(x, y) \in \mathbb{R}^{2}: x, y \geq 0\right\}$ be the cone in A. Define a function $\varphi: P \rightarrow P$ by $\varphi(a)=$ ka for all $a \in P$, where $k \in(0,1)$. Then $\varphi$ is a comparison function. 


\section{Main Results}

We start this section by introducing the notations of $\alpha$-admissible and $\alpha$ - $\varphi$-contractive mappings.

Definition 2.1. Let $A$ be a Banach algebra, $P$ be a cone in $A$ and $X$ be a nonempty set. Let $T: X \rightarrow X$ and $\alpha: X \times X \times X \rightarrow P$. We say that $T$ is $\alpha$-admissible if

$$
x, y \in X, \quad \alpha(x, y, a) \succeq e \forall a \in X \Longrightarrow \alpha(T x, T y, a) \succeq e \forall a \in X .
$$

Example 2.2. Let $A=\mathbb{R}^{2}$ and for each $\left(x_{1}, x_{2}\right) \in A,\left\|\left(x_{1}, x_{2}\right)\right\|=\left|x_{1}\right|+\left|x_{2}\right|$. The multiplication in $A$ is defined by $\left(x_{1}, x_{2}\right) \cdot\left(y_{1}, y_{2}\right)=\left(x_{1} y_{1}, x_{1} y_{2}+x_{2} y_{1}\right)$. Then $A$ is a Banach algebra with unit $e=(1,0)$. Let $P=[0, \infty) \times[0, \infty)$ be the cone in $A$. Let $X=\mathbb{R}^{2}$ and define the mappings $T: X \rightarrow X$ and $\alpha: X \times X \times X \rightarrow P$ by

$$
\begin{gathered}
T\left(x_{1}, x_{2}\right)=\left(x_{1}^{3}, \sin x_{2}\right) \text { for all }\left(x_{1}, x_{2}\right) \in X ; \text { and } \\
\alpha\left(\left(x_{1}, x_{2}\right),\left(y_{1}, y_{2}\right),\left(a_{1}, a_{2}\right)\right)=\left\{\begin{array}{l}
\left(2+x_{1} y_{1}+\frac{a_{1}}{\left|a_{1}\right|+\left|a_{2}\right|}, 1+\left|x_{2} y_{2}\right|-\frac{a_{1}}{\left|a_{1}\right|+\left|a_{2}\right|}\right) \text { if } x_{1}, y_{1} \geq 0 \\
0 \text { otherwise. }
\end{array}\right.
\end{gathered}
$$

Then the mapping $T$ is $\alpha$-admissible.

Example 2.3. Let $A=\mathbb{R}^{2}$ and for each $\left(x_{1}, x_{2}\right) \in A,\left\|\left(x_{1}, x_{2}\right)\right\|=\left|x_{1}\right|+\left|x_{2}\right|$. The multiplication in $A$ is defined by $\left(x_{1}, x_{2}\right) \cdot\left(y_{1}, y_{2}\right)=\left(x_{1} y_{1}, x_{1} y_{2}+x_{2} y_{1}\right)$. Then $A$ is a Banach algebra with unit $e=(1,0)$. Let $P=[0, \infty) \times[0, \infty)$ be the cone in $A$. Let $X=[0, \infty) \times[0, \infty), r \in \mathbb{N}$ and define the mappings $T: X \rightarrow X$ and $\alpha: X \times X \times X \rightarrow P$ by

$$
\begin{gathered}
T x=x^{r} \quad \text { for all } x \in X ; \text { and } \\
\alpha(x, y, a)= \begin{cases}\left(\frac{x_{1}+y_{1}}{a_{1}+1}, a_{2}\right) \quad \text { if } x_{1}+y_{1} \geq a_{1}+1 ; \\
\left(\frac{a_{1}+1}{x_{1}+y_{1}}, a_{2}\right) \quad \text { if } a_{1}+1 \geq x_{1}+y_{1}>0 \\
\theta \quad \text { otherwise }\end{cases}
\end{gathered}
$$

where $x=\left(x_{1}, x_{2}\right), y=\left(y_{1}, y_{2}\right), a=\left(a_{1}, a_{2}\right) \in X$. Then the mapping $T$ is $\alpha$-admissible.

Definition 2.4. Let $(X, d)$ be a cone 2-metric space over Banach algebras $A$ and $T: X \rightarrow X$ be a given mapping. We say that $T$ is a $\varphi$-contractive mapping if there exists a comparison function $\varphi$ such that

$$
d(T x, T y, a) \preceq \varphi(d(x, y, a)) \text { for all } x, y, a \in X
$$


Definition 2.5. Let $(X, d)$ be a cone 2-metric space over Banach algebras $A$ and $T: X \rightarrow X$ be a given mapping. We say that $T$ is an $\alpha$ - $\varphi$-contractive mapping if there exist a function $\alpha: X \times X \times X \rightarrow P$ and a comparison function $\varphi$ such that

$$
\alpha(x, y, a) d(T x, T y, a) \preceq \varphi(d(x, y, a)) \text { for all } x, y, a \in X
$$

Remark 2.6. If we define the function $\alpha: X \times X \times X \rightarrow P$ by $\alpha(x, y, a)=e$ for all $x, y, a \in X$, then it is easy to see that every $\varphi$-contractive mapping on a cone 2-metric space is an $\alpha$ - $\varphi$ contractive mapping.

Example 2.7. Let $X=[0, \infty), A=C^{1}([0,1], \mathbb{R})$ be the Banach algebra with point wise multiplication and the norm defined by $\|x(t)\|=\|x(t)\|_{\infty}+\left\|x^{\prime}(t)\right\|_{\infty}$. Let $P=\{x(t) \in$ $C^{1}([0,1], \mathbb{R}): x(t) \geq 0$ for all $\left.t \in[0,1]\right\}$ be the solid cone in A. Define a mapping $d: X \times$ $X \times X \rightarrow P$ by $d(x, y, z)=\rho e^{t}$ for all $x, y, z \in X$, where $\rho=\min \{|x-y|,|y-z|,|z-x|\}$. Then $(X, d)$ is a cone 2-metric space over Banach algebras A. Define a mapping $\alpha: X \times X \times X \rightarrow$ $P$ by

$$
\alpha(x, y, a)= \begin{cases}1, & \text { if } a>x, y ; \text { and } \rho=|x-y| ; \\ 0, & \text { otherwise. }\end{cases}
$$

Define a mapping $T: X \rightarrow X$ by $T x=\frac{x}{1+x}$ for all $x \in X$. Then $T$ is an $\alpha$ - $\varphi$-contractive mapping with $\varphi(\phi(t))=\frac{\phi(t)}{1+\phi(t)}$ for all $\phi \in P$.

Definition 2.8. Let $(X, d)$ be a cone 2-metric space over Banach algebras $A$ and $T: X \rightarrow X$ be a mapping. Then, $T$ said to be continuous at point $x \in X$, if for every sequence $\left\{x_{n}\right\}$ in $X$ we have $T x_{n} \rightarrow T x$ as $n \rightarrow \infty$, whenever $x_{n} \rightarrow x$ as $n \rightarrow \infty$. T said to be continuous if it is continuous at every point of $X$.

Next, we state an existence result of the fixed point of a continuous $\alpha-\varphi$-contractive mapping on cone 2-metric spaces.

Theorem 2.9. Let $(X, d)$ be a complete cone 2-metric space over Banach algebras $A$ and $T: X \rightarrow X$ be an $\alpha-\varphi$-contractive mapping satisfying the following conditions:

(i) $T$ is $\alpha$-admissible;

(ii) there exists $x_{0} \in X$ such that $\alpha\left(x_{0}, T^{r} x_{0}, a\right) \succeq$ e for all $a \in X, r \in \mathbb{N}$;

(iii) $T$ is continuous.

Then $T$ has a fixed point, that is, there exists $x^{*} \in X$ such that $T x^{*}=x^{*}$.

Proof. Let $x_{0} \in X$ be such that $\alpha\left(x_{0}, T^{r} x_{0}, a\right) \succeq e$ for all $a \in X, r \in \mathbb{N}$. Define a sequence $\left\{x_{n}\right\}$ in $X$ by

$$
x_{n+1}=T x_{n} \quad \text { for all } n \in \mathbb{N} .
$$


Therefore, $\alpha\left(x_{0}, x_{r}, a\right) \succeq e$ for all $a \in X, n \in \mathbb{N}$. By $\alpha$-admissibility of $T$ we have $\alpha\left(T x_{0}, T x_{r}, a\right)=$ $\alpha\left(x_{1}, x_{r+1}, a\right) \succeq e$ for all $a \in X, n \in \mathbb{N}$. By induction we obtain

$$
\alpha\left(x_{n}, x_{r+n}, a\right) \succeq e \text { for all } a \in X, n \in \mathbb{N} .
$$

If $x_{n}=x_{n+1}$ for some $n \in \mathbb{N}$, then $x^{*}=x_{n}$ is a fixed point of $T$. Assume that $x_{n} \neq$ $x_{n+1}$ for all $n \in \mathbb{N}$.

Since $T$ is an $\alpha$ - $\varphi$-contractive mapping, using (3) and Remark 1.2 we obtain: for all $a \in X$

$$
\begin{aligned}
d\left(x_{n}, x_{n+1}, a\right) & =d\left(T x_{n-1}, T x_{n}, a\right) \\
& \preceq \alpha\left(x_{n-1}, x_{n}, a\right) d\left(T x_{n-1}, T x_{n}, a\right) \\
& \preceq \varphi\left(d\left(x_{n-1}, x_{n}, a\right)\right) .
\end{aligned}
$$

Using the properties of $\varphi$, by induction we obtain that

$$
d\left(x_{n}, x_{n+1}, a\right) \preceq \varphi^{n}\left(d\left(x_{0}, x_{1}, a\right)\right) \text { for all } n \in \mathbb{N}, a \in X .
$$

For $c \gg \theta$, we can choose $n_{0} \in \mathbb{N}$ and $\delta>0$ such that

$$
\begin{gathered}
c-\varphi(c)+\{u \in A:\|u\|<\delta\} \subset \operatorname{int} P, \quad\left\|\varphi^{n}\left(d\left(x_{0}, x_{1}, a\right)\right)\right\|<\delta \\
\quad \text { and } \varphi^{n}\left(d\left(x_{0}, x_{1}, a\right)\right) \ll c-\varphi(c) \text { for all } n>n_{0}, a \in X .
\end{gathered}
$$

Therefore, by Lemma 1.3, (4) and the above inequality we obtain

$$
d\left(x_{n}, x_{n+1}, a\right) \ll c-\varphi(c) \preceq c \text { for all } n>n_{0}, a \in X .
$$

We shall show that the sequence $\left\{x_{n}\right\}$ is a Cauchy sequence. Then, for $n>n_{0}$, using (3) we have

$$
\begin{aligned}
d\left(x_{n}, x_{n+2}, a\right) & \preceq d\left(x_{n}, x_{n+2}, x_{n+1}\right)+d\left(x_{n}, x_{n+1}, a\right)+d\left(x_{n+1}, x_{n+2}, a\right) \\
& =d\left(x_{n}, T x_{n+1}, T x_{n}\right)+d\left(x_{n}, x_{n+1}, a\right)+d\left(T x_{n}, T x_{n+1}, a\right) \\
& \preceq d\left(x_{n}, T x_{n+1}, T x_{n}\right)+d\left(x_{n}, x_{n+1}, a\right)+\alpha\left(x_{n}, x_{n+1}, a\right) d\left(T x_{n}, T x_{n+1}, a\right) \\
& \preceq \varphi\left(d\left(x_{n}, x_{n+1}, x_{n}\right)\right)+d\left(x_{n}, x_{n+1}, a\right)+\varphi\left(d\left(x_{n}, x_{n+1}, a\right)\right) \\
& \ll c-\varphi(c)+\varphi(c) \\
& =c .
\end{aligned}
$$

Similarly, for $n>n_{0}$ using (3) we obtain

$$
\begin{aligned}
d\left(x_{n}, x_{n+3}, a\right) & \preceq d\left(x_{n}, x_{n+3}, x_{n+1}\right)+d\left(x_{n}, x_{n+1}, a\right)+d\left(x_{n+1}, x_{n+3}, a\right) \\
& =d\left(x_{n}, T x_{n+2}, T x_{n}\right)+d\left(x_{n}, x_{n+1}, a\right)+d\left(T x_{n}, T x_{n+2}, a\right) \\
& \preceq d\left(x_{n}, T x_{n+2}, T x_{n}\right)+d\left(x_{n}, x_{n+1}, a\right)+\alpha\left(x_{n}, x_{n+2}, a\right) d\left(T x_{n}, T x_{n+2}, a\right) \\
& \preceq \varphi\left(d\left(x_{n}, x_{n+2}, x_{n}\right)\right)+d\left(x_{n}, x_{n+1}, a\right)+\varphi\left(d\left(x_{n}, x_{n+2}, a\right)\right) \\
& \ll c-\varphi(c)+\varphi(c) \\
& =c .
\end{aligned}
$$


By induction, we obtain

$$
d\left(x_{n}, x_{n+r}, a\right) \ll c \text { for all } r \in \mathbb{N} \text { and } n \geq n_{0} .
$$

Thus, $\left\{x_{n}\right\}$ is a Cauchy sequence in the cone 2-metric space. Since $(X, d)$ is complete, there exists $x^{*} \in X$ such that $x_{n} \rightarrow x^{*}$ as $n \rightarrow \infty$. We shall show that $x^{*}$ is a fixed point of $T$.

From the continuity of $T$, it follows that $x_{n+1}=T x_{n} \rightarrow T x^{*}$ as $n \rightarrow \infty$. In view of Remark 1.8 we obtain $x^{*}=T x^{*}$, that is, $x^{*}$ is a fixed point of $T$.

In the next theorem, we replace the continuity of $T$ by another hypothesis which does not depend on the nature of $T$.

Theorem 2.10. Let $(X, d)$ be a complete cone 2-metric space over Banach algebras A and $T: X \rightarrow X$ be an $\alpha-\varphi$-contractive mapping such that the following conditions are satisfied:

(i) $T$ is $\alpha$-admissible;

(ii) there exists $x_{0} \in X$ such that $\alpha\left(x_{0}, T^{r} x_{0}, a\right) \succeq$ e for all $a \in X, r \in \mathbb{N}$;

(iii) if $\left\{x_{n}\right\}$ is a sequence in $X$ such that $\alpha\left(x_{n}, x_{n+1}, a\right) \succeq$ e for all $n \in \mathbb{N}$ and $x_{n} \rightarrow x \in X$ as $n \rightarrow \infty$, then $\alpha\left(x_{n}, x, a\right) \succeq$ e for all $n \in \mathbb{N}$.

Then $T$ has a fixed point, that is, there exists $x^{*} \in X$ such that $T x^{*}=x^{*}$.

Proof. Following the arguments to those in the proof of Theorem 2.9, we obtain that $\left\{x_{n}\right\}$ is a Cauchy sequence in $X$. By completeness of $(X, d)$, there exists $x^{*} \in X$ such that $x_{n} \rightarrow x^{*}$ as $n \rightarrow \infty$. On the other hand, from (3) and the hypothesis (iii), we have

$$
\alpha\left(x_{n}, x^{*}, a\right) \succeq e, \text { for all } a \in X, n \in \mathbb{N} .
$$

Now, using (2), (6) and Proposition 1.2, we obtain

$$
\begin{aligned}
d\left(T x^{*}, x^{*}, a\right) \preceq & d\left(T x^{*}, x^{*}, T x_{n}\right)+d\left(T x^{*}, T x_{n}, a\right)+d\left(T x_{n}, x_{n}, a\right) \\
\preceq & \alpha\left(x_{n}, x^{*}, x^{*}\right) d\left(T x_{n}, T x^{*}, x^{*}\right)+\alpha\left(x_{n}, x^{*}, a\right) d\left(T x_{n}, T x^{*}, a\right) \\
& +d\left(x_{n+1}, x^{*}, a\right) \\
\preceq & \varphi\left(d\left(x_{n}, x^{*}, x^{*}\right)\right)+\varphi\left(d\left(x_{n}, x^{*}, a\right)\right)+d\left(x_{n+1}, x^{*}, a\right) \\
= & \varphi\left(d\left(x_{n}, x^{*}, a\right)\right)+d\left(x_{n+1}, x^{*}, a\right)
\end{aligned}
$$

for all $a \in X$. Suppose, $c \in \operatorname{int} P$ be given. Since $x_{n} \rightarrow x^{*}$ as $n \rightarrow \infty$, there exists $n_{0} \in \mathbb{N}$ such that $d\left(x_{n}, x^{*}, a\right) \ll c / 2, d\left(x_{n+1}, x^{*}, a\right) \ll c / 2$ for all $n>n_{0}, a \in X$. So, by the properties of the function $\varphi$ we have $\varphi\left(d\left(x_{n}, x^{*}, a\right)\right) \preceq \varphi(c / 2) \ll c / 2$ for all $n>n_{0}, a \in X$. Therefore, it follows from the above inequality that

$$
d\left(T x^{*}, x^{*}, a\right) \ll \frac{c}{2}+\frac{c}{2}=c \text { for all } n>n_{0}, a \in X .
$$

Using Lemma 1.3 we obtain $d\left(T x^{*}, x^{*}, a\right)=\theta$ for all $a \in X$, that is, $T x^{*}=x^{*}$. Thus, $x^{*}$ is a fixed point of $T$. 
Example 2.11. Let $X=\{(x, 0),(0, x): 0 \leq x \leq 1\}$ and $A=\mathbb{R}^{2}$ be the Banach algebra with the norm $\left\|\left(x_{1}, x_{2}\right)\right\|=\left|x_{1}\right|+\left|x_{2}\right|$ and the multiplication defined by $\left(x_{1}, x_{2}\right)\left(y_{1}, y_{2}\right)=$ $\left(x_{1} x_{2}, x_{2} y_{1}+y_{2} x_{1}\right)$. Then $e=(1,0)$ is the unit of $A$. Let $P=\left\{\left(x_{1}, x_{2}\right) \in \mathbb{R}^{2}: x_{1}, x_{2} \geq 0\right\}$ be the cone in $A$. Define a mapping $d: X \times X \times X \rightarrow P$ by: $d\left(\alpha_{1}, \alpha_{2}, \alpha_{3}\right)=d_{1}\left(\beta_{1}, \beta_{2}\right)$ for all $\alpha_{1}, \alpha_{2}, \alpha_{3} \in X$, where $\beta_{1}, \beta_{2} \in\left\{\alpha_{1}, \alpha_{2}, \alpha_{3}\right\}$ are such that $\left\|\beta_{1}-\beta_{2}\right\|=\min \left\{\| \alpha_{1}-\right.$ $\left.\alpha_{2}\|,\| \alpha_{2}-\alpha_{3}\|,\| \alpha_{3}-\alpha_{1} \|\right\}$ and

$$
\begin{aligned}
& d_{1}((x, 0),(y, 0))=\left(\frac{5}{4}|x-y|,|x-y|\right) \\
& d_{1}((0, x),(0, y))=\left(|x-y|, \frac{3}{4}|x-y|\right) \\
& \left.d_{1}((x, 0),(0, y))=d_{1}((0, y),(x, 0))=\left(\frac{5}{4} x+y, x+\frac{3}{4} y\right)\right) .
\end{aligned}
$$

Then $(X, d)$ is a complete cone 2 -metric space. For some $a, b \in \mathbb{Q}$ such that $a>\frac{4}{5}, b>\frac{4}{3}$, define the mapping $T: X \rightarrow X$ by

$$
T(x, 0)=\left\{\begin{array}{ll}
\left(0, \frac{x}{a}\right), & \text { if } x \in \mathbb{Q}_{[0,1]} ; \\
\left(\frac{1}{\sqrt{2}}, 0\right), & \text { otherwise, }
\end{array} \quad T(0, x)= \begin{cases}\left(\frac{x}{b}, 0\right), & \text { if } x \in \mathbb{Q}_{[0,1]} ; \\
\left(0, \frac{1}{\sqrt{3}}\right), & \text { otherwise }\end{cases}\right.
$$

where $\mathbb{Q}_{[0,1]}=\mathbb{Q} \cap[0,1]$. Denote by $S_{0}$ the set of all sequences in $\mathbb{Q}_{[0,1]}$ converging to 0 , that is, $S_{0}=\left\{\left\{x_{n}\right\}: x_{n} \in \mathbb{Q}_{[0,1]}, n \in \mathbb{N}, x_{n} \rightarrow 0\right.$ as $\left.n \rightarrow \infty\right\}$; and define $\alpha: X \times X \times X \rightarrow P$ by

$$
\begin{aligned}
& \alpha\left(\left(x_{n}, 0\right),\left(0, y_{n}\right),\left(a_{1}, a_{2}\right)\right)=\alpha\left(\left(0, x_{n}\right),\left(y_{n}, 0\right),\left(a_{1}, a_{2}\right)\right)=e, \\
& \alpha\left(\left(x_{n}, 0\right),\left(y_{n}, 0\right),\left(a_{1}, a_{2}\right)\right)=\alpha\left(\left(0, x_{n}\right),\left(0, y_{n}\right),\left(a_{1}, a_{2}\right)\right)=e,
\end{aligned}
$$

where $\left\{x_{n}\right\},\left\{y_{n}\right\} \in S_{0}$ and for all $\left(a_{1}, a_{2}\right) \in X$; and $\alpha(x, y, a)=\theta=(0,0)$ for all other values of $x, y, a \in X$. Then the mapping $T$ is a $\alpha$ - $\varphi$-contractive mapping with $\varphi\left(x_{1}, x_{2}\right)=k\left(x_{1}, x_{2}\right)$, $\max \left\{\frac{4}{5 a}, \frac{4}{3 b}\right\} \leq k<1$. Note that, if we choose $x_{n}=\frac{1}{a^{n}}$ for all $n \in \mathbb{N}$, then $\left\{x_{n}\right\} \in S_{0}$ and $\alpha\left(\left(\frac{1}{a^{n}}, 0\right), T^{r}\left(\frac{1}{a^{n}}, 0\right),\left(a_{1}, a_{2}\right)\right)=e$ for all $\left(a_{1}, a_{2}\right) \in X$ and $r \in \mathbb{N}$. Therefore, the condition (ii) of Theorem 2.10 is satisfied. Now, it is easy to see that the mapping $T$ is an $\alpha$-admissible mapping and the condition (iii) of Theorem 2.10 is satisfied.

Thus, all the conditions of Theorem 2.10 are satisfied. Therefore, we can apply Theorem 2.10 to conclude the existence of fixed point of $T$. Indeed, the set of fixed points of $T$,

$$
\operatorname{Fix}(T)=\left\{(0,0),\left(\frac{1}{\sqrt{2}}, 0\right),\left(0, \frac{1}{\sqrt{3}}\right)\right\} .
$$

It is clear from the above example that the fixed point of the class of mappings considered in the previous theorem may not be unique. To assure the uniqueness of the fixed point, we will consider the following hypothesis.

(H): For all $x, y \in X$, there exists $z \in X$ such that $\alpha(x, z, a) \succeq e$ and $\alpha(y, z, a) \succeq e$ for all $a \in X$. 
Theorem 2.12. Adding condition $(H)$ to the hypotheses of Theorem 2.9 (resp. Theorem 2.10) we obtain uniqueness of the fixed point of $T$.

Proof. Suppose that $x^{*}$ and $y^{*}$ are two distinct fixed point of $T$. From (H), there exists $z \in X$ such that

$$
\alpha\left(x^{*}, z, a\right) \succeq e \text { and } \alpha\left(y^{*}, z, a\right) \succeq e \text { for all } a \in X .
$$

The $\alpha$-admissibility of $T$ with the above inequality yields

$$
\alpha\left(T^{n} x^{*}, T^{n} z, a\right)=\alpha\left(x^{*}, T^{n} z, a\right) \succeq e \text { and } \alpha\left(T^{n} y^{*}, T^{n} z, a\right)=\alpha\left(y^{*}, T^{n} z, a\right) \succeq e
$$

for all $a \in X$ and $n \in \mathbb{N}$. Since $T$ is an $\alpha-\varphi$-contractive mapping using the above inequality and Remark 1.2 we obtain

$$
\begin{aligned}
d\left(x^{*}, T^{n} z, a\right) & =d\left(T x^{*}, T^{n} z, a\right)=d\left(T x^{*}, T T^{n-1} z, a\right) \\
& \preceq \alpha\left(x^{*}, T^{n-1} z, a\right) d\left(T x^{*}, T T^{n-1} z, a\right) \\
& \preceq \varphi\left(d\left(x^{*}, T^{n-1} z, a\right)\right)
\end{aligned}
$$

for all $a \in X$. Repetition of the above process and the properties of $\varphi$ give:

$$
d\left(x^{*}, T^{n} z, a\right) \preceq \varphi^{n}\left(d\left(x^{*}, z, a\right)\right) \text { for all } a \in X, n \in \mathbb{N} .
$$

Since $\lim _{n \rightarrow \infty} \varphi^{n}\left(d\left(x^{*}, z, a\right)\right)=\theta$, by Lemma 1.3, for every $c \in \operatorname{int} P$ there exists $n_{0} \in \mathbb{N}$ such that $d\left(x^{*}, T^{n} z, a\right) \ll c$ for all $a \in X, n>n_{0}$. Therefore, $T^{n} z \rightarrow x^{*}$ as $n \rightarrow \infty$. With a similar process one can obtain $T^{n} z \rightarrow y^{*}$ as $n \rightarrow \infty$, and so, by in view of Remark 1.8 we obtain $x^{*}=y^{*}$. This contradiction proves the uniqueness of fixed point.

The following corollary is a fixed point result for a $\varphi$-contractive mapping on cone 2-metric spaces.

Corollary 2.13. Let $(X, d)$ be a complete cone 2-metric space over Banach algebras A and $T: X \rightarrow X$ be a $\varphi$-contractive mapping. Then $T$ has a unique fixed point, that is, there exists unique $x^{*} \in X$ such that $T x^{*}=x^{*}$.

Proof. Define the function $\alpha: X \times X \times X \rightarrow P$ by $\alpha(x, y, a)=e$ for all $x, y, a \in X$. Then, it is easy to see that all the conditions of Theorem 2.12 are satisfied and we conclude the existence of unique fixed point of $T$.

The following corollary is an improved version of the Ran and Reurings [1] fixed point theorem in cone 2-metric spaces.

Corollary 2.14. Let $(X, \sqsubseteq)$ be a partial ordered set and there exists a cone 2-metric $d$ such that $(X, d)$ is a complete cone 2-metric space over Banach algebras $A$. Let $T: X \rightarrow X$ be a mapping such that the following conditions are satisfied: 
(i) there exists a cone comparison function $\varphi$ such that

$$
d(T x, T y, a) \preceq \varphi(d(x, y, a))
$$

for all $x, y, a \in X$ with $x \sqsubseteq y$;

(ii) $T$ is nondecreasing, that is, $x \sqsubseteq y \Longrightarrow T x \sqsubseteq T y$ for all $x, y \in X$;

(iii) There exists $x_{0} \in X$ such that $x_{0} \sqsubseteq T x_{0}$;

(iv) $T$ is continuous.

Then $T$ has a fixed point, that is, there exists $x^{*} \in X$ such that $T x^{*}=x^{*}$.

Proof. Define the mapping $\alpha: X \times X \times X \rightarrow P$ by

$$
\alpha(x, y, a)= \begin{cases}e, & \text { if } x \sqsubseteq y ; \\ \theta, & \text { otherwise }\end{cases}
$$

for all $a \in X$. Then, the proof follows from Theorem 2.9.

The following corollary is an improved version of the Nieto and Rodríguez-López's [7] fixed point theorem in cone 2-metric spaces.

Corollary 2.15. Let $(X, \sqsubseteq)$ be a partial ordered set and there exists a cone 2-metric $d$ such that $(X, d)$ is a complete cone 2-metric space over Banach algebras A. Let $T: X \rightarrow X$ be a mapping such that the following conditions are satisfied:

(i) there exists a cone comparison function $\varphi$ such that

$$
d(T x, T y, a) \preceq \varphi(d(x, y, a))
$$

for all $x, y, a \in X$ with $x \sqsubseteq y$;

(ii) $T$ is nondecreasing, that is, $x \sqsubseteq y \Longrightarrow T x \sqsubseteq T y$ for all $x, y \in X$;

(iii) There exists $x_{0} \in X$ such that $x_{0} \sqsubseteq T x_{0}$;

(iv) if $\left\{x_{n}\right\}$ is a sequence in $X$ such that $x_{n} \sqsubseteq x_{n+1}$ for all $n$ and $x_{n} \rightarrow x \in X$ as $n \rightarrow \infty$, then $x_{n} \sqsubseteq x$ for all $n \in \mathbb{N}$.

Then $T$ has a fixed point, that is, there exists $x^{*} \in X$ such that $T x^{*}=x^{*}$.

Proof. Define the mapping $\alpha: X \times X \times X \rightarrow P$ by

$$
\alpha(x, y, a)= \begin{cases}e, & \text { if } x \sqsubseteq y ; \\ \theta, & \text { otherwise }\end{cases}
$$

for all $a \in X$. Then, the proof follows from Theorem 2.9. 


\section{References}

[1] A.C.M. RAN, M.C.B. REURINGS, A fixed point theorem in partially ordered sets and some applications to matrix equations, Proc. Amer. Math. Soc., 132 (2003) 1435-1443.

[2] B. Samet, C. Vetro, P. Vetro, Fixed point theorems for $\alpha$ - $\psi$-contractive type mappings, Nonlinear Analysis, 75 (2012) 2154-2165.

[3] B. SINGH, S. JAIN, P. BHAGAT, Cone 2-metric space and fixed point theorem of contractive mappings, Comment. Math., 52(2), 143-151 (2012).

[4] C. Di BARI, P. VETRO, $\varphi$-Pairs and common fixed points in cone metric space, Rendiconti del Circolo Matematico di Palermo, 57 (2008) 279-285.

[5] H. ÇAKAlli, A. SÖnmeZ, Ç. GEnç, On an equivalence of topological vector space valued cone metric spaces and metric spaces, Appl. Math. Lett., 25 (2012) 429-433.

[6] H. LiU AND S.-Y. XU, Cone metric spaces with Banach algebras and fixed point theorems of generalized Lipschitz mappings, Fixed Point Theory Appl., 2013, 2013:320.

[7] J.J. NiETo, R. RodRíGuEz-LóPEZ, Contractive mapping theorems in partially ordered sets and applications to ordinary differential equations, Order, 22 (2005) 223-239.

[8] J.J. Nieto, R. RodrígueZ-LópeZ, Existence and uniqueness of fixed point in partially ordered sets and applications to ordinary differential equations, Acta Math. Sin. (Engl. Ser.), 23 (2007) 2205-2212.

[9] L.G. HuAng, X. ZHANG, Cone metric spaces and fixed point theorems of contractive mappings, J. Math. Anal. Appl., 332 (2007) 1468-1476.

[10] S.GÄHLER, 2-metricsche Räume und ihre topologische strukture, Math. Nachr. 26 (1963) 115-148.

[11] S.GÄHLER, Uber die Uniformisierbarkeit 2-metricsche Räume, Math. Nachr. 28 (1965) 235244.

[12] S.GÄHLER, Zur geometric 2-metricsche Räume, Revne Roumaine der Mathem. Pures et Appliques, 11 (1966) 665-667.

[13] S. RADENOVIĆ, B.E. RHOADES, Fixed point theorem for two non-self mappings in cone metric spaces, Comput. Math. Appl., 57 (2009) 1701-1707.

[14] S. XU, S. RADENOVIĆ, Fixed point theorems of generalized Lipschitz mappings on cone metric spaces over Banach algebras without assumption of normality, Fixed Point Theory Appl., 2014, 2014:102.

[15] T. WANG, J. YIN, Q. YAN, Fixed point theorems on cone 2-metric spaces over Banach algebras and an application, Fixed Point Theory Appl., (2015) 2015:204.

[16] W. Rudin, Functional Analysis, McGraw-Hill, New York, NY, USA, 2nd edition, 1991.

[17] W.S. DU, A note on cone metric fixed point theory and its equivalence, Nonlinear Anal., 72(5), (2010) 2259-2261.

[18] Y. Feng, W. MaO, The equivalence of cone metric spaces and metric spaces, Fixed Point Theory, 11(2) (2010) 259-264. 
[19] Z. KAdELBURG, S. RADENOVIĆ, V. RAKoČEVIĆ, A note on the equivalence of some metric and cone metric fixed point results, Appl. Math. Lett., 24 (2011) 370-374.

[20] Z. Kadelburg, M. Pavlović, S. Radenović, Common fixed point theorems for ordered contractions and quasi-contractions in ordered cone metric spaces, Comput. Math. Appl., 59 (2010) 3148-3159. 